\title{
Strength in Numbers: Exploiting the space between single crystal oscillation and serial femtosecond crystallography
}

\author{
D. Axford, S. Horrell, R. L. Owen \\ Diamond Light Source, Harwell Oxford, Didcot, OX11 ODE, UK \\ danny.axford@diamond.ac.uk
}

Right from its initial conception, the micro-focus beamline I24 at Diamond Light Source has looked beyond the assumption that an experimenter's structural question would be answerable with a single, well diffracting, cryo-cooled sample. A multi-crystal approach to data collection has become a modus operandi. Initially attention was focused on small volume and weakly diffracting samples that would typically receive a destructive X-ray dose before complete and redundant data could be recorded. To help tackle this requirement, pipelines for rapid collection and intelligent merging of thin wedges of data from multiple crystals have been developed. Additionally, Serial Synchrotron crystallography (SSX) has become a core activity, with the intention of probing structural dynamics obtainable within protein crystals at room temperature [1,2]. This brings the requirement for many thousands of crystals, each contributing only a tiny proportion of the final dataset and providing a challenge in terms of collection and processing. I present latest results from SSX and multi-crystal experiments, describe the tools available for users of the beamline and consider optimum methods for successful many-crystal experiments.

[1] Ebrahim et al. (2019). IUCrJ. 6, 543-551.

[2] Rabe et al. (2020). IUCrJ. 7, 901-912.

Keywords: macromolecular crystallography; multi-crystal; serial crystallography; SSX; high-throughput 